\title{
Lateral Damage Identification in RC Slabs by Several Tomographic Approaches with Rainy Induced Elastic Waves
}

\author{
Tomoki Shiotani ${ }^{1, *}$ - Katsufumi Hashimoto ${ }^{1}$ - Hisafumi Asaue ${ }^{1}$ - Takahiro Nishida ${ }^{1}$ \\ - Hidefumi Takamine ${ }^{2}$ - Kazuo Watabe ${ }^{2}$ - Masato Fukuda ${ }^{3}$ \\ ${ }^{1}$ Department of Civil \& Earth Resources Engineering, Kyoto University, Japan \\ ${ }^{2}$ Toshiba Corporation, Japan \\ ${ }^{3}$ West Nippon Expressway Company Limited, Japan
}

Efficient inspection techniques for ageing infrastructures are in great demand. In this study, rainy induced acoustic activity, which has so far been treated as a nuisance event for acoustic monitoring, is ambitiously utilized to identify damages of reinforced concrete (RC) slabs. Specifically, in-situ acoustic emission (AE) measurements of RC bridge slabs are conducted for a week. First, internal damages of the slabs are evaluated by both of $A E$ activity and elastic wave velocity by means of $A E$ monitoring and $A E$ tomography. As for some representative locations showing each different damage estimated by the $A E$ activity and the velocity, core samples are retrieved for the verification. In addition, $A E$ activity induced by rain droplets in a short period of minutes are identified by an AE source location algorithm followed by computation on attenuation rate distribution. Through the evaluations, it was found that the distribution of AE sources induced by precipitation could reflect internal damage of RC slabs i.e., dense areas of source locations imply the intact or minor damage, while sparse areas of source locations suggest serious damage of $R C$ decks. With this finding, the prompt decision making if the decks shall be replaced, repaired or left, which could not so far be readily implemented by other inspection techniques, would be reasonably conducted.

Keywords: acoustic emission, damage identification, raindrops, lateral damage, RC slabs

Highlights

- $A E$ source distribution due to precipitation of rain induced elastic wave excitation, the serious damage as to be developed lateral cracks could be estimated for the low-density area of the distribution.

- The results of $A E$ tomography with rainy induced $A E$ activity was compatible to the results of $A E$ tomography with using secondary $A E$ activity generated inside of the $R C$ deck.

- $\quad$ Attenuation rate distribution by means of AE tomography in this panel was also successfully computed to show the presence of lateral crack in the deck.

- It was also clarified that the distribution of attenuation rates by means of AE tomography could identify the damaged zones inside concrete.

\section{INTRODUCTION}

It is generally recognized that preventive and proactive maintenance works are necessary for such important infrastructure as bridges and tunnels. For reinforced concrete (RC) members, essential issues include establishing a maintenance system with the appropriate measures prior to the extensive damage and failure.

As a result of budgetary restrictions, preventive and proactive maintenance of infrastructure are desired, and thus inspections by non-destructive testing (NDT) methods providing early damage information must be applied. In terms of the damage assessment and estimation of repair and retrofit recovery in concrete structures, in addition to current NDT, innovative methods must be established.

The authors are thus studying tomography techniques based on elastic waves and acoustic emission (AE) to visualize three dimensional internal defects in concrete. In AE tomography, past reports can be found in literatures [1] and [2]; however, as they did not consider diversion of wave propagation paths (hereafter referred to as ray paths), few successful results can be obtained. In consideration of the diversion of the ray paths, the basic analytical procedure and the applicability of these techniques have already been reported as elastic-wave tomography [3] and AE tomography [4] by authors.

Through the tomography technique, internal conditions are obtained using elastic wave parameters such as amplitudes and elastic wave velocities. This technique has been developed in the field of seismology and geophysics [5] to [7] as well as ultrasonic [8] to [10]. In this study, elastic wave velocity and attenuation rate are [11] used as the parameter. In elastic wave tomography, both the location of the excitation and the excitation time are known, whereas they are unknown for AE tomography. Specifically, the tomography can evaluate the elastic wave velocity 
and the attenuation rate in each set-element over the structure, which are theoretically associated with the modulus of elasticity. Because of the presence of such internal defects as cracks and voids, the elastic waves are reflected, diffracted, and scattered inside media with anomaly such as voids and cracks. The effect results in a decrease in elastic-wave velocity or energy. Thus, it can reasonably be assumed that the areas exhibiting lower elastic-wave velocity and higher attenuation rate correspond to those of serious deterioration. Accordingly, the distribution of wave velocities can be referred to as a good indicator of the internal condition of concrete structures.

\section{AE ACTIVITY MEASUREMENT AND AE TOMOGRAPHY}

\subsection{Estimation of Wave Velocity Distribution}

In in-situ RC Bridge decks, secondary AE activities, generated by friction among existing crack interfaces under traffic loads can be measured. Therefore, the evolutional damage area can be visualized and specified when plotting $\mathrm{AE}$ sources through the measurement period; while the tomography can estimate internal velocity distributions of the structure, of which the velocity value can correspond to the quality of the concrete. The basic analysis procedure is shown as follows. Some AE sensors can record arrival time of elastic wave when the wave is generated by an AE source or artificial excitations such as hammering. After each arrival time is obtained, the propagation velocity through the propagation path of elastic wave is calculated by both of the distance from the excitation point to the receive point and $T_{o b s}$ (observed propagation time) which is obtained by Eq. (1):

$$
T_{o b s}=T_{o}-T_{s},
$$

where $T_{s}$ is the excitation time and $T_{o}$ is the arrival time. In the algorithm of the tomography, the inverse of velocity which is specifically referred to as the "slowness" is given as an initial parameter of each element as shown in Fig. 1. Next, $T_{\text {cal }}$ (theoretical propagation time) is obtained, which is the total of the propagation time calculated by the slowness and the distance in each element (refer Eq. (2)), where $s_{j}$ is the slowness of each element and $l_{j}$ is the length crossing each element. $\Delta T$ is defined by observed propagation time $\left(T_{o b s}\right)$ and theoretical propagation time $\left(T_{c a l}\right)$ as shown in Eq. (3):

$$
\begin{gathered}
T_{c a l}=\sum_{j} s_{j} \times l_{j}, \\
\Delta T=T_{o b s}-T_{c a l},
\end{gathered}
$$

Then the slowness in each element is revised in order to reduce $\Delta T$. The slowness correction amount and the revised slowness are obtained by Eqs. (4) and (5), respectively:

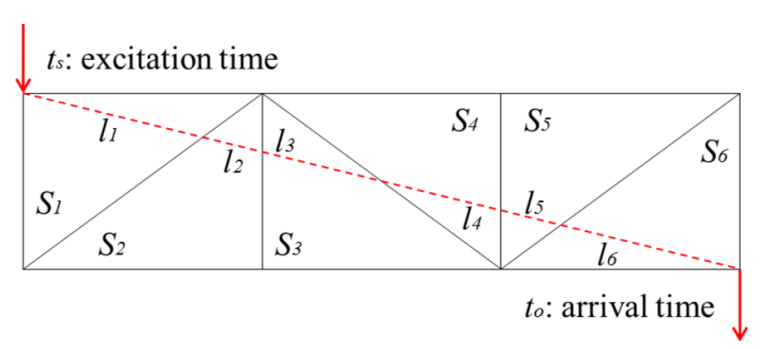

Fig. 1. Slowness for calculation of propagation time

$$
\left[\begin{array}{c}
\Delta s_{1} \\
\Delta s_{2} \\
\vdots \\
\Delta s j
\end{array}\right]=\left[\begin{array}{c}
\sum_{i} \frac{\Delta T_{i} \times l_{i 1}}{L_{i}} / \sum_{i} l_{i 1} \\
\sum_{i} \frac{\Delta T_{i} \times l_{i 2}}{L_{i}} / \sum_{i} l_{i 2} \\
\vdots \\
\sum_{i} \frac{\Delta T_{i} \times l_{i j}}{L_{i}} / \sum_{i} l_{i j}
\end{array}\right],
$$

where $L_{i}$ is the total distance of the wave in the $i^{\text {th }}$ element. The iteration calculation from Eqs. (4) to (5) enables to obtain the accurate slowness and finally the velocity in each element corresponding to the observed propagation time of multiple waves over the structure, resulting in forming the tomogram of the elastic wave velocity over the target area. Through these steps, velocity distributions were determined in the structure. It is noted that in the AE tomography, the AE source identification and velocity distributions are both unknown and therefore they are calculated iteratively using AE tomography algorithm [12].

\subsection{AE Measurement and Analysis}

A real RC bridge deck was selected as a study target which has deterioration such as rebar corrosion due to salt attack and fatigue failure due to repeated mobile loads. The AE measurement was carried out with $\mathrm{AE}$ sensors, set on the bottom side of RC bridge decks. Resonant frequency of the $\mathrm{AE}$ sensor is $30 \mathrm{kHz}$, arranged on the RC deck as shown in Fig. 2.

Thickness of the RC bridge decks is $235 \mathrm{~mm}$. Serious damage could be expected for the deck as large cracks with water leakage trace has been already observed. Threshold value of location uncertainty (LUCY) is set on $300 \mathrm{~mm}$, about half space of two 
adjacent sensors in this study. LUCY means source location accuracy and is the root-mean-square of the difference between calculated and observed distances between the source and the sensor [13].

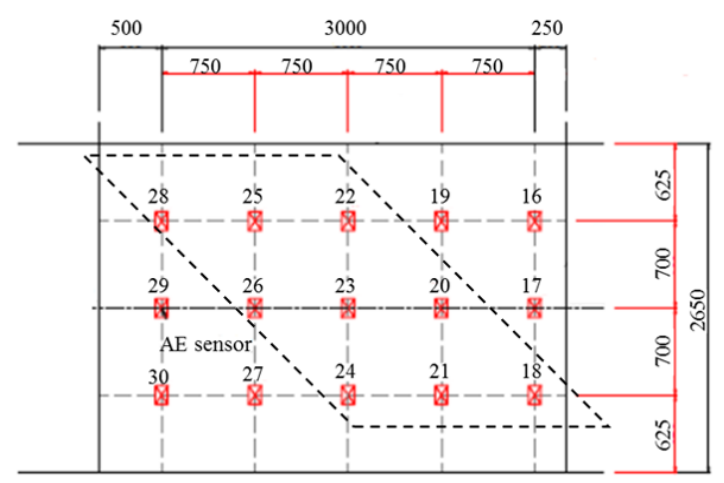

Fig. 2. Sensor arrangements for $A E$ measurement on in-situ $R C$ bridge decks

Dotted lines of parallelogram in Fig. 2 shows the cut-off specimen to study precise evaluation with the AE tomography, where three-dimensional analysis is carried out to estimate velocity distributions inside of the panel. AE sensor array for the tomography analysis is also shown in Fig. 3.

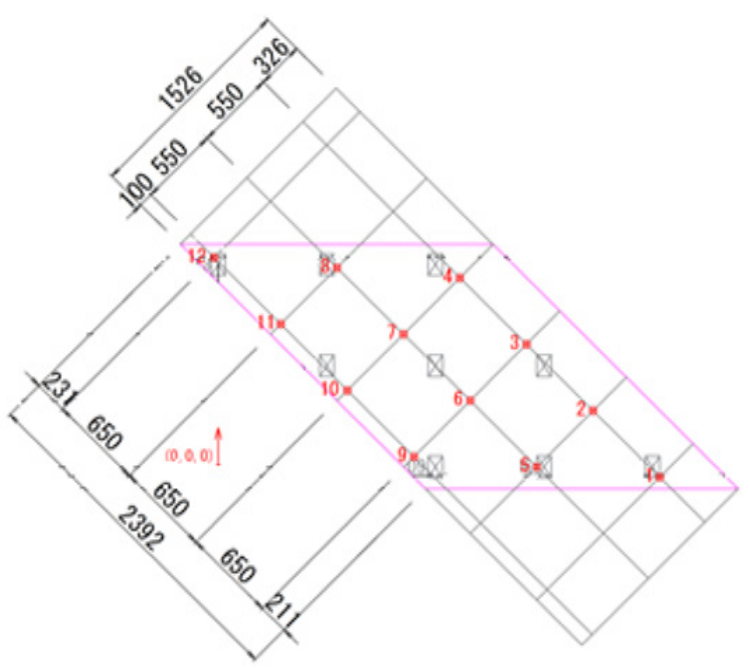

Fig. 3. Sensor arrangement in random hammering for $A E$ tomography (Unit: $\mathrm{mm}$ )

\subsection{AE Tomography}

12 sensors of $60 \mathrm{kHz}$ resonance were newly set on the bottom of panel. Random excitations with a hammer of a $\phi 11 \mathrm{~mm}$ curvature edge were carried out for exciting elastic waves on the top surface of the panel. Appropriate input sources were carefully selected on the following conditions: the case that the number of hits for an $\mathrm{AE}$ event is more than five by one hammering and the other is that LUCY is under 300 $\mathrm{mm}$. As the results, the number of input sources for the tomography were 51 . Results of AE tomography are shown in Fig. 4.

a)

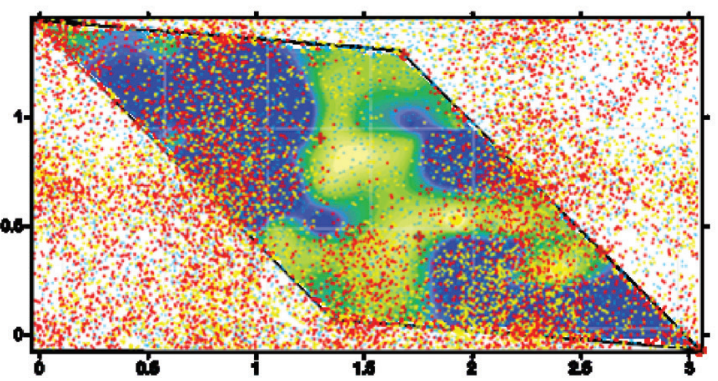

b)

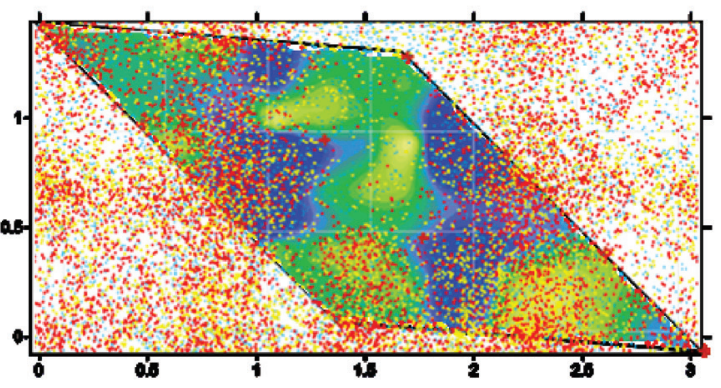

c)

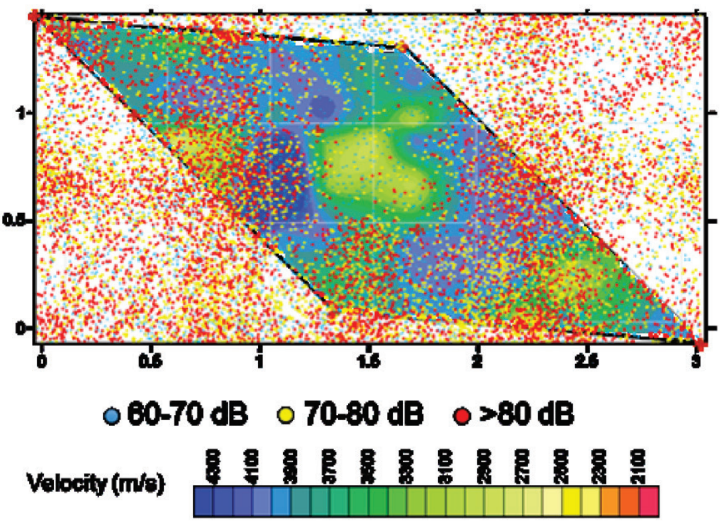

Fig. 4. Velocity distributions from the top to the bottom of RC deck with $A E$ source location classified with peak amplitude of the first arrival of the AE hit; a) top layer, b) middle layer, c) bottom layer

In general, for the $\mathrm{AE}$ activity, the more intensive $\mathrm{AE}$ activity is obtained, the more damage than of lees is expected in the media. As for the velocity, the area showing large velocity namely $4000 \mathrm{~m} / \mathrm{s}$ suggests intact condition whereas small velocity of less than $3000 \mathrm{~m} / \mathrm{s}$ roughly implies serious damage. As shown in Fig. 4, however, the area showing small velocity exhibits less AE activity while the area showing large velocity denotes the intensive $\mathrm{AE}$ activity. Some representative locations were subsequently selected, 
and cores at those locations had been sampled and compared with the results both from the AE activity and the velocity. As a result, the followings were found: the area exhibiting small velocity with less $\mathrm{AE}$ activity showed serious damage existed inside of the deck, whereas intensive $\mathrm{AE}$ activity with large velocity suggested in damage progress condition, and less $\mathrm{AE}$ activity with large velocity implied intact condition [4].

\section{RAIN-INDUCED AE ACTIVITY}

\subsection{AE Activity with Rain Drops}

AE hits per half an hour for 135 hours is shown in Fig. 5. An intensive $\mathrm{AE}$ activity were acquired from 120 to 130 hours. Besides, rain droplets are also known as a factor generating $\mathrm{AE}$ activity when it impacts on a solid material [14]. As considered factors of AE activity in the panel, some of the AE activity, shown in Fig. 5, might be generated as the resultant impacts of rain droplets on the surface of RC deck.
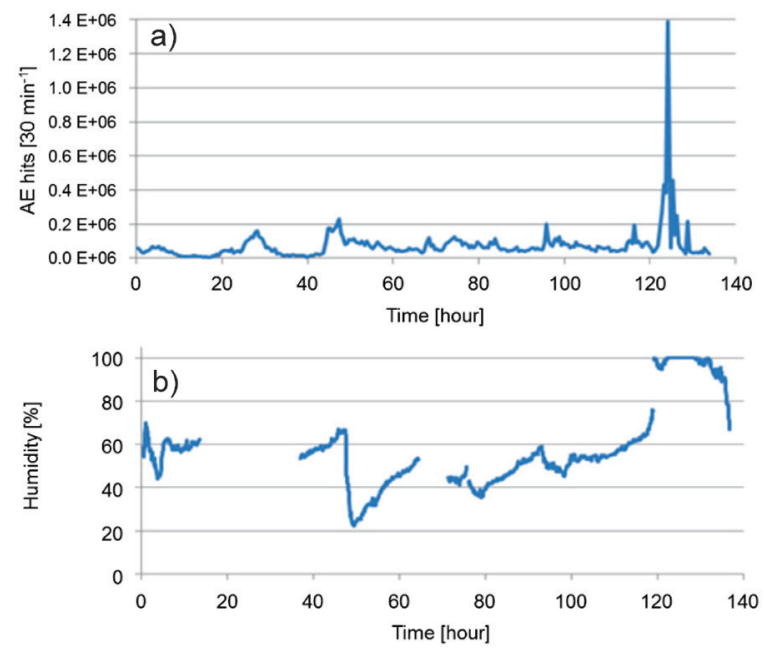

Fig. 5. a) $A E$ activities and b) relative humidity during a measurement period

To clarify the fact of precipitation, relative humidity at the bridge is shown as in Fig. 5 as well as $\mathrm{AE}$ activity. After 120 hours, humidity increased to $100 \%$ accompanied with the high AE activity. In the weather record, precipitation was also confirmed at that period. Thus, the intensive AE activities is considered to be caused by heavy rainfall. It indicates that the impacts of raindrops on the road surface caused AE activity, and some AE events reached the sensors attached on the bottom surface of the deck. Those concentrated AE activities was neither to correlate to the traffic load nor to be considered as a result of existing cracks inside the bridge decks. Therefore, those signals are in general considered not to reflect the deterioration of the deck, leading to be treated as noise. However, a unique distribution of $\mathrm{AE}$ sources induced by these rain droplets was found, the following discussion would be developed to inspect the bridge decks.

\subsection{Source Location of Rainy Induced AE Hits}

The AE data was extracted during the intensive rain and analyzed for the source locations. Fig. 6 shows the source locations of the measured panel. Here, the source locations considered to have low reliability has been filtered out. Enough amounts of AE sources for evaluating the distribution of $\mathrm{AE}$ sources were obtained only for 700 seconds. In the figure, a lowdensity area surrounded by dashed lines can be confirmed on the panel. As the random AE events by the precipitation shall be resulted in uniform distribution of $\mathrm{AE}$ sources over the bridge deck; however, non-uniformity of the source distribution exhibiting an empty area of the source distribution is obvious, and therefore this low-density area is attributed from extremely high attenuation of AE wave propagation in the propagation media, suggesting the suspect to be heavily deteriorated. In consideration of velocity distribution as well as visual inspection by actually excavated core samples it has been concluded that the area of less AE activity accorded well to the laterally damaged or cracked locations. Accordingly, it was found that rainy induced AE activity had a potential to determine the area of serious deterioration in the concrete decks.

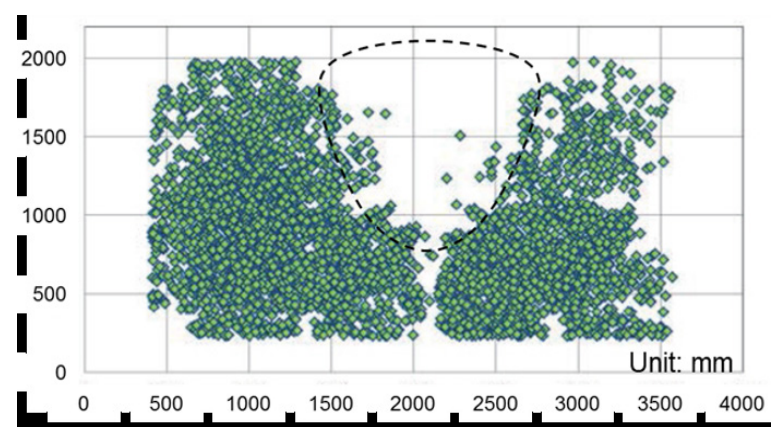

Fig. 6. Result of $A E$ source location analysis

Specifically, by calculating the AE source locations, the density of the $\mathrm{AE}$ sources would reveal the damage condition inside the deck. In addition, since all $\mathrm{AE}$ sources generated by raindrops are on the same surface of the road surface, instead of $3 \mathrm{D}$, 


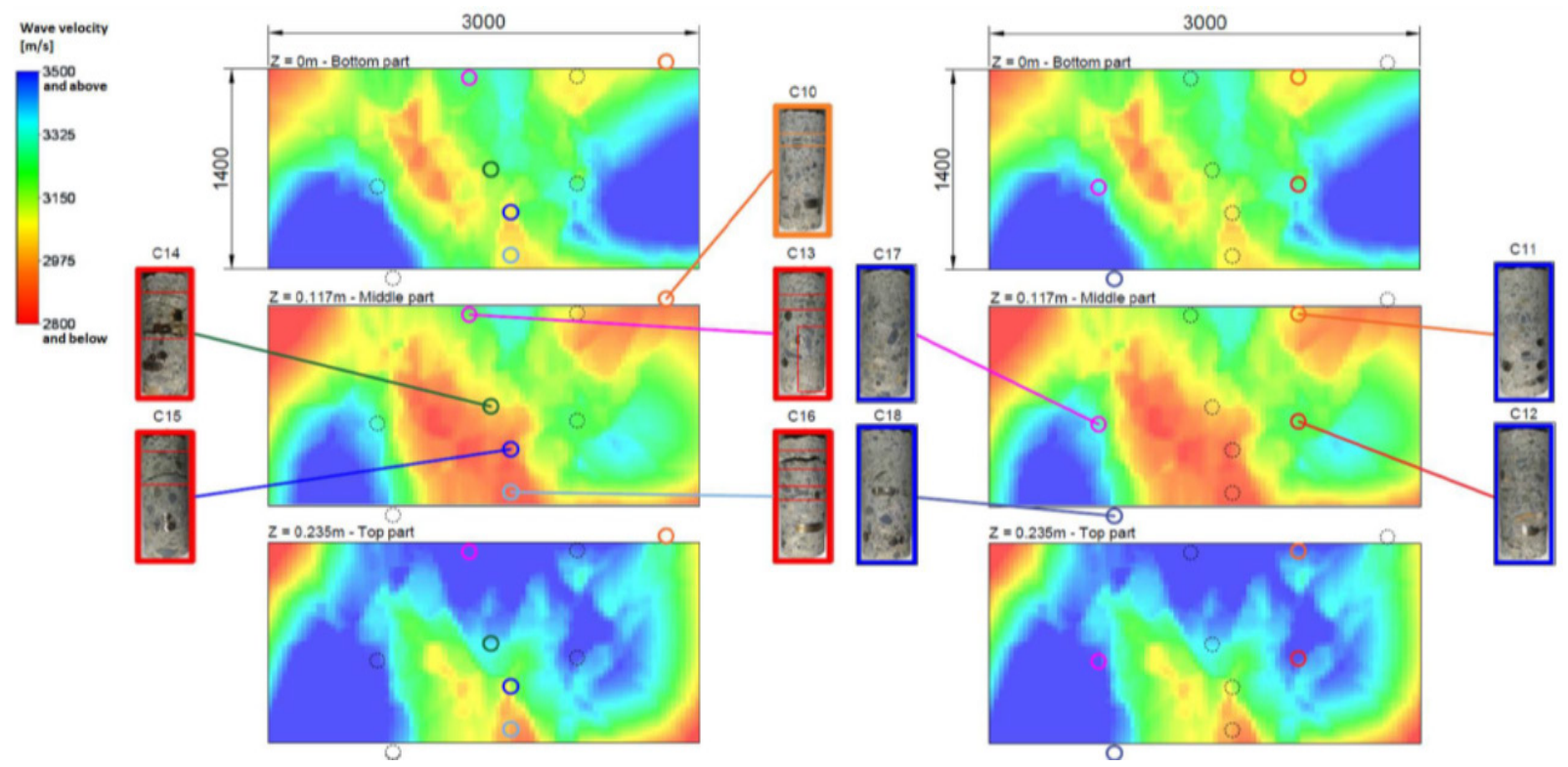

Fig. 7. Comparison between the tomograms and the core samples

simple 2D source locations can be readily conducted. As the short-term heavy rain could generate a large amount of $\mathrm{AE}$ hits on the road, providing reasonable interpretation on the specification of the internal damage, the damage assessment of the decks induced by rain droplets has thus a great potential to realize insitu damage inspection with great efficiency.

\subsection{AE Tomography with Rainy Induced AE Activity}

As expected, a sufficient number of rainy induced $\mathrm{AE}$ events has been obtained, the wave velocity distribution by means of $\mathrm{AE}$ tomography in this panel has also been conducted using rainy induced $\mathrm{AE}$ sources and the results are presented in Fig. 7 with core samples taken out from the deck to verify the results. The deck after core excavation can be found as in Fig. 8 .

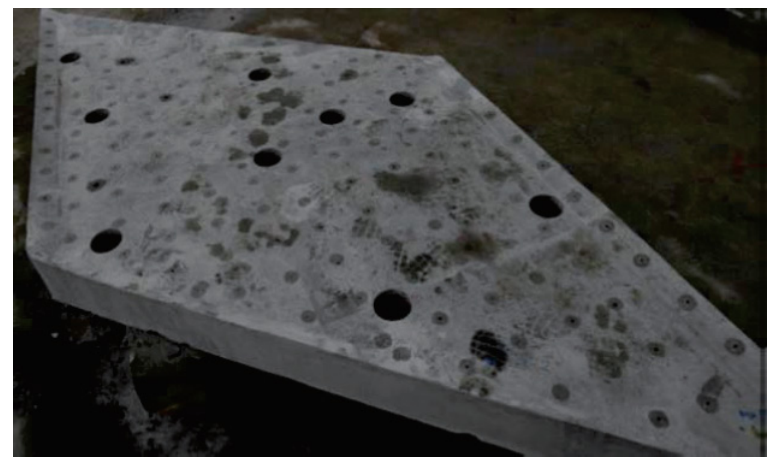

Fig. 8. Positions of core samples
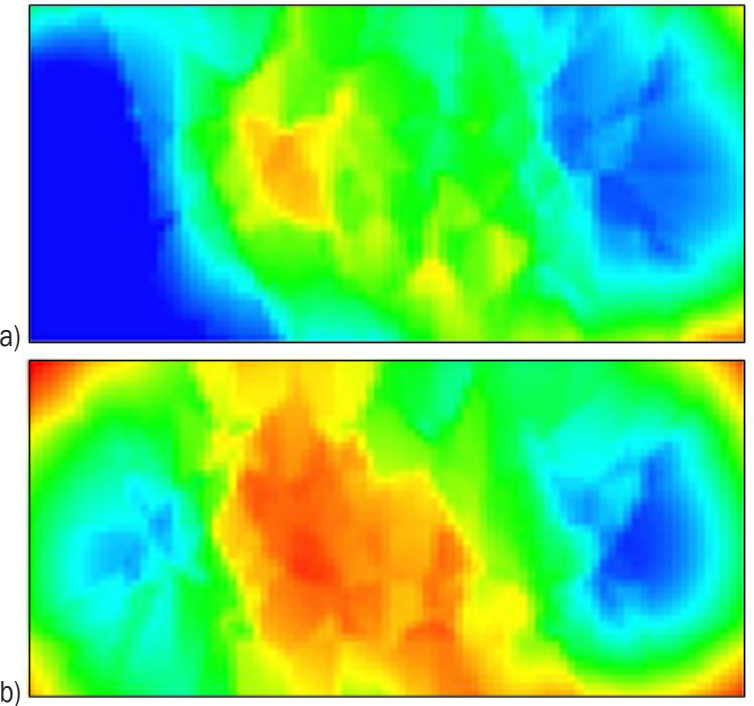

c)

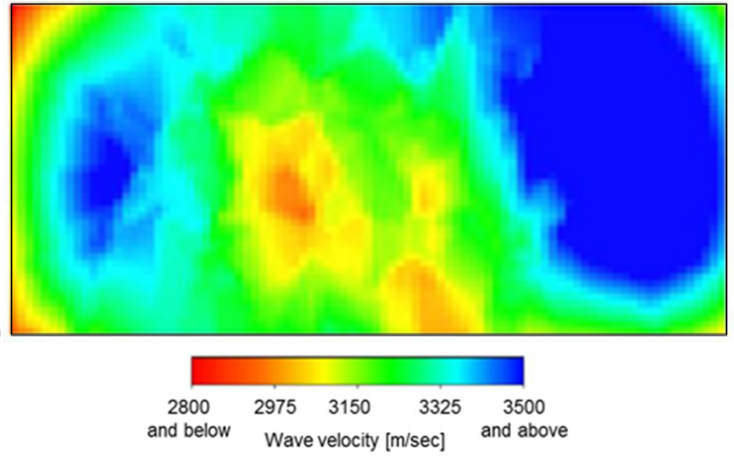

Fig. 9. $A E$ tomography results with internal $A E$ activity: a) $z=0 \mathrm{~mm}$ (bottom surface), b) $Z=117 \mathrm{~m}$ (middle layer), c) $Z=235 m$ (top surface) 
As shown in Fig. 7, the lateral damage was observed in the cores showing small velocity and vice versa. Nevertheless, this is not exactly true for the core samples $\mathrm{C} 11$ and $\mathrm{C} 13$ which are both located at the border between a low velocity zone and a medium velocity zone. These small inaccuracies may simply be caused by the fact that the fineness of the mesh determining elements of analysis does not allow for such a precise evaluation or secondary damage produced during cut-off work.

The velocity distributions obtained by using internal $\mathrm{AE}$ events is shown in Fig. 9 for the comparison to that obtained by using rainy induced $\mathrm{AE}$ events as found in Fig. 7. The tomogram obtained by means of rainy induced $\mathrm{AE}$ events, is almost compatible to that obtained by the internal $\mathrm{AE}$ events, suggesting $\mathrm{AE}$ events produced by the precipitation in a short-term provides a reasonable tomogram result, being equivalent when using secondary $\mathrm{AE}$ events in a long-term.

\subsection{Attenuation Tomography}

As the computation for the elastic wave ray-trace algorithm considering all potential detours of elastic waves takes up much time, hereafter a straight line of $\mathrm{P}$-wave propagation is assumed in the tomography analysis via the attenuation-rate distribution to identify the lateral cracks in $\mathrm{RC}$ slab. The data processing flow is shown as in Fig. 10 so as to extract meaningful AE events. As in the figure, first, the source locations of all $\mathrm{AE}$ events are used as input, where a constant wave velocity $[\mathrm{m} / \mathrm{s}]$ and attenuation-rate $[\mathrm{dB} / \mathrm{m}]$ across the tested member is assumed for calculation. Second, the amplitude and the excitation time at the source of the considered AE event are estimated on the basis of the arrival times and the amplitudes recorded by the sensors. Third, the attenuation rates along all straight ray-paths between the source and the receiving sensors are computed.

For each AE event, the peak amplitude of the elastic wave at the source is unknown. Consequently, it can be approximated to calculate the attenuation rate along the considered wave paths. In the attenuation tomography algorithm, they are generally estimated from a relation represented in Fig. 11 (the distance in logarithmic scale is used in general). The peak amplitude of the signal recorded by each sensor is plotted as a function of the distance between the source and the sensors. A linear regression between the peak amplitude of the elastic wave and the distance from the source is computed. The peak amplitude at the source is referred to as equal to the value for the case that the distance is equal to zero.

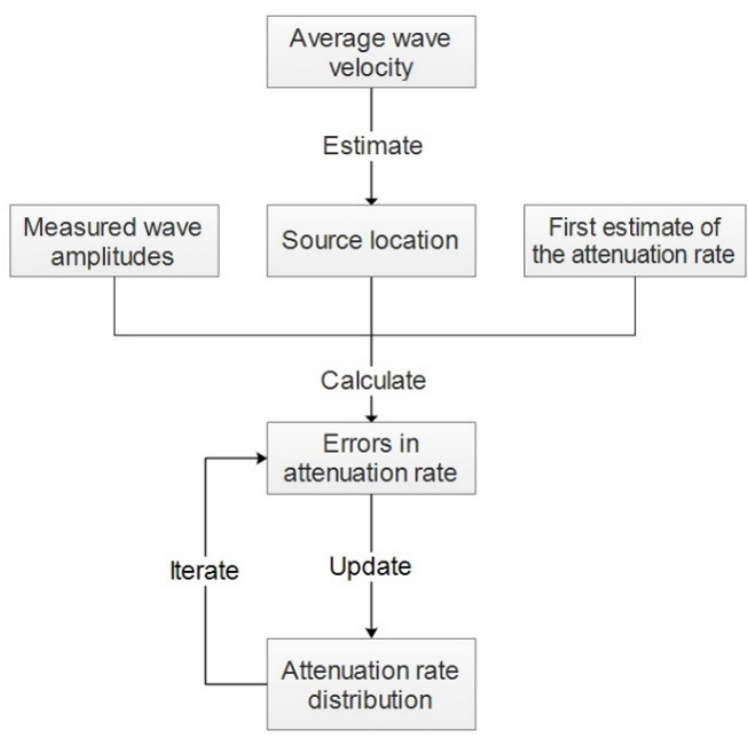

Fig. 10. Flow chart to compute attenuation tomography

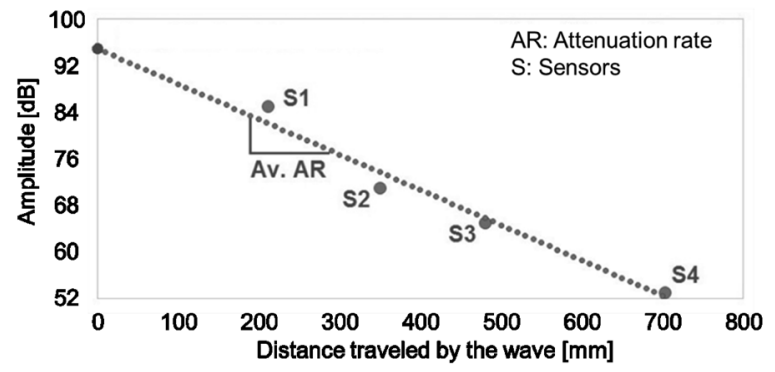

Fig. 11. Estimation of the peak amplitude of the elastic wave at the source

Tomography computation is carried out based on elastic wave parameters with the attenuation rate in this paper. In the tomography based on the attenuation, the area of interest and analysis must be divided into mesh elements characterized by their own attenuation rates. Then, a first estimate on distribution of the attenuation rates is to be provided as input. By comparing those values along each wave path to its calculated value of the assumed distribution, the SIRT algorithm [15] could lead to proper distribution of the attenuation rates. The measured travel time and attenuation rate along each ray path is estimated from Eq. (6).

$$
A R_{\text {measured }, i}=\frac{A_{\text {source }}-A_{\text {sensor }, i},}{\sum_{j}^{N_{i}} d_{i, j}},
$$

where $A R_{\text {measured }, i}$ is measured average attenuation rate along the wave path from the source to the $i^{\text {th }}$ sensor, 
$0:$ Un-cracked $\times:$ Cracked

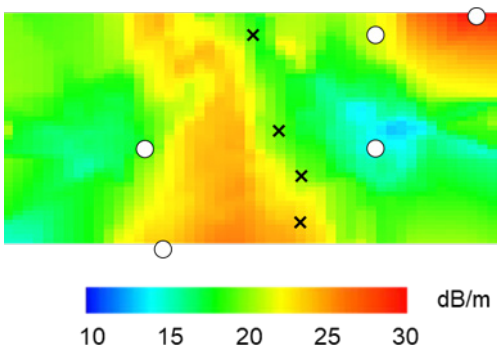

$0:$ Un-cracked $\times:$ Cracked

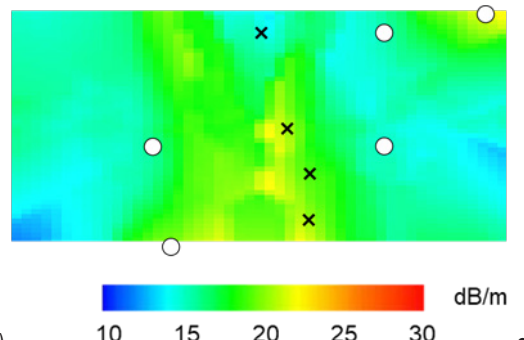

$0:$ Un-cracked $\times:$ Cracked

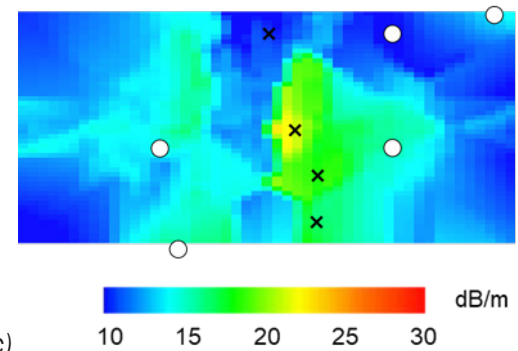

Fig. 12. Attenuation rate distributions; $a) z=0 \mathrm{~mm}$ (bottom surface), b) $Z=117 \mathrm{~m}$ (middle layer), $c$ ) $Z=235 \mathrm{~m}$ (top surface)

$A_{\text {source }}$ estimated peak amplitude of the elastic wave associated to the considered AE event at its source, $A_{\text {receiver, } i}$ peak amplitude of the elastic wave measured at the $i^{\text {th }}$ sensor, $N_{i}$ mesh number of elements crossed by the wave path from the source to the $i^{\text {th }}$ sensor, and $d_{i, j}$ length of the wave path from the source to the $i^{\text {th }}$ sensor in the $j^{\text {th }}$ element.

Second, the attenuation rate along each wave path based on distribution of the attenuation rates in the mesh elements is computed by Eq. (7).

$$
A R_{\text {calculated }, i}=\frac{\sum_{j}^{M} A R_{j} \cdot d_{i, j}}{\sum_{j}^{M} d_{i, j}}
$$

where $A R_{\text {calculated, } i}$ is calculated average attenuation rate along the wave path from the source to the $i^{\text {th }}$ sensor $[\mathrm{dB} / \mathrm{m}], A R_{j}$ attenuation rate in the $j^{\text {th }}$ element $[\mathrm{dB} / \mathrm{m}]$, and $M$ mesh number of elements crossed by the ray path from the source to the $i^{\text {th }}$ sensor.

Afterwards, the difference between the measured and the calculated attenuation rates is calculated for each wave path by using Eq. (8).

$$
\Delta A R_{i}=A R_{\text {measured }, i}-A R_{\text {estimated }, i}
$$

In a similar manner to Eq. (1), the differences of the attenuation rates on all the wave paths are estimated by Eq. (9).

$$
\Delta A R_{j}=\frac{\sum_{i=1}^{N} \Delta A R_{i} \cdot d_{i, j}}{\sum_{i=1}^{N} d_{i, j}},
$$

where $N$ is the number of wave paths crossing the $j^{\text {th }}$ element. The attenuation rate in each element is then updated with Eq. (10).

$$
A R_{j, \text { updated }}=A R_{j}-\triangle A R_{j}
$$

The procedure from Eq. (7) through Eq. (10) is repeated until the convergence is reached.
The attenuation rate distribution by means of $\mathrm{AE}$ tomography, as explained above, in this panel has been successfully computed in Fig. 12 with conditions' observation by sampled cores. The lateral cracks were located at $150 \mathrm{~mm}$ or less from the surface of the deck (mostly in the top layer) by visual inspection of the cored samples [16]. Therefore, it is reasonably understood that attenuation rate in top layer are lower than bottom layer. The soundness of concrete and the presence of lateral crack roughly correspond to the attenuation rate distribution. In this study, the attenuation rate inside the core samples presenting large lateral cracks are generally higher than $20 \mathrm{~dB} / \mathrm{m}$ at least over a certain part of their depth.

\section{CONCLUSIONS}

The elastic waves generated by the rain droplets could be an AE activity contributing to the AE tomography. The results of AE tomography with rainy induced AE activity in a short term was compatible to the results of AE tomography with using secondary AE activity generated inside of the concrete deck in a long term. With the AE source distribution due to precipitation, the serious damage as to be developed lateral cracks could be estimated for the low-density area of the distribution. It is also clarified that the distribution of attenuation rates by means of AE tomography could identify the damaged zones inside concrete. With these findings, the prompt decision making if the decks shall be replaced, repaired or left, which could not so far be readily implemented by other inspection techniques, would be reasonably conducted.

\section{ACKNOWLEDGEMENTS}

A part of this study was commissioned and supported by New Energy and Industrial Technology Development Organization (NEDO). 


\section{REFERENCES}

[1] Katsuyama, K., Seto, M., Kiyama, T., Utagawa M. (1992). Three dimensional AE tomography for image processing of the deteriorated material. Safety Engineering, JSSE, p. 321-326. (in Japanese)

[2] Schubert, F. (2004). Basic principles of acoustic emission tomography, DGZfP, Proceedings BB 90- CD, EWGAE 2004, Lecture 58.

[3] Shiotani, T., Aggelis, D. G., Momoki, S. (2009). Elastic wave validation of large concrete structures repaired by means of cement grouting. Construction and Building Materials, vol. 23, p. 2647-2652, D0I:10.1016/j.conbuildmat.2009.01.005.

[4] Asuae, H., Shiotani, T., Nishida, T., Watabe, K., Miyata, H. (2016). Applicability of AE tomography for accurate damage evaluation in actual RC bridge deck. Structural Faults \& Repair Conference, no. 1743 (CD-ROM).

[5] ISRM (1988). Suggested Methods for seismic testing within and between boreholes. Seismic Testing Suggested Methods, p. 449-472.

[6] Saito, H. (1989). Travel times and raypaths of first arrival seismic waves: Computation method based on Huygens' Principle, 59th Annual International Meeting, Society of Exploration Geophysicists, Expanded Abstracts, p. 244-247.

[7] Saito, H. (1992). Characteristics of the first arrival travel time curves obtained by crosshole seismic measurements. Proceedings of the 86 th SEGJ Conference, p. 88-93.

[8] Kak, A.C., Slaney, M. (1998). Principles of Computerized Tomographic Imaging, IEEE Press, New York.
[9] Schechter, R. S., Mignogna, R. B. and Delsanto, P. P. (1996). Ultrasonic tomography using curved ray paths obtained by wave propagation simulations on a massively parallel computer. Journal of Acoustic Society of America, vol. 100, no. 4, p. 2103-2111, Dol:10.1121/1.417920.

[10] Mignogna, R.B., Delsanto, P.P. (1996). A parallel approach to acoustic tomography. Journal of Acoustic Society of America, vol. 99, no. 4, p. 2142-2147, Dol:10.1121/1.415401.

[11] Chai, H.K., Momoki, S., Kobayashi, Y., Aggelis, D.G., Shiotani, T. (2011). Tomographic reconstruction for concrete using attenuation of ultrasound, NDT\&E International, vol. 44, no. 2, p. 206-215, D0l:10.1016/j.ndteint.2010.11.003.

[12] Kobayashi, Y., Shiotani, T. (2012). Seismic tomography with estimation of source location for concrete structure. Structural Faults and Repair 2012, (CD-ROM).

[13] Hamstad, M.A. (2007). Acoustic emission source location in a thick steel plate using lamb modes. Journal of Acoustic Emission, vol. 25, p. 194-214.

[14] Shiotani, T. (2006). Evaluation of long-term stability for rock slope by means of acoustic emission. NDT\&E International, vol.39, p. 217-228, D0l:10.1016/j.ndteint.2005.07.005.

[15] Tranmpert, J., Leveque, J.J. (1990). Simultaneous iterative reconstruction technique: physical interpretation based on the generalized least squares solution. Journal of Geophysical Research, vol. 95, no. B8, p. 12553-12559, D0l:10.1029/ JB095iB08p12553.

[16] Takamine, H., Watabe, K., Miyata, H., Asaue, H., Nishida, T., Shiotani, T. (2016). Efficient damage inspection of deteriorated RC bridge deck with rain-induced AE activity. Proceedings of IAES-23, IIIAE 2016, Kyoto and ICAE 8, p. 231-236. 